\title{
Carriage of multiple Streptococcus pneumoniae capsular types is frequent among children with invasive pneumococcal disease
}

\author{
Sofia Félix ${ }^{1}$. Desirée Henares ${ }^{2} \cdot$ Carmen Muñoz-Almagro $^{2,3,4} \cdot$ Raquel Sá-Leão $^{1}$ (D) \\ Received: 13 November 2020 / Accepted: 17 March 2021 / Published online: 2 April 2021 \\ (C) The Author(s), under exclusive licence to Springer-Verlag GmbH Germany, part of Springer Nature 2021
}

\begin{abstract}
Streptococcus pneumoniae (pneumococcus) is a human pathogen that colonizes the nasopharynx. We investigated serotype distribution in paired invasive and nasopharyngeal samples obtained from 57 children during invasive pneumococcal disease. Of 39 nasopharyngeal samples positive for pneumococci, $46.2 \%$ contained a serotype different from the one causing disease. This study reports a high frequency of pneumococcal multiple serotype carriage in children with invasive pneumococcal disease. Whether multiple serotype carriage is important for the onset and progress to pneumococcal infection warrants further investigation.
\end{abstract}

Keywords Streptococcus pneumoniae · Invasive pneumococcal disease $\cdot$ Children · Multiple serotype carriage $\cdot$ Co-colonization

\section{Background}

Streptococcus pneumoniae, or the pneumococcus, is a frequent colonizer of the nasopharynx, particularly during childhood. Occasionally, the pneumococcus migrates to normally sterile body sites and this, through a single cell bottleneck, may result in infections such as invasive pneumonia, bacteremia, and meningitis [1].

The major virulence factor of pneumococcus is the capsule, which is immunogenic. One-hundred capsular types (or serotypes) have been described to date [2]. Multi-valent pneumococcal conjugate vaccines (PCV) target a subset $(7,10$, or 13) of these serotypes.

Simultaneous carriage of multiple pneumococcal serotypes, or co-colonization, has been found to occur in 20-30\% of young healthy children in Europe [3, 4]. Competition between

Raquel Sá-Leão

rsaleao@itqb.unl.pt

1 Laboratory of Molecular Microbiology of Human Pathogens, Instituto de Tecnologia Química e Biológica António Xavier/ Universidade Nova de Lisboa (ITQB/NOVA), Oeiras, Portugal

2 Molecular Microbiology Department, Instituto de Recerca Pediatrica/University Hospital Sant Joan de Déu, Barcelona, Spain

3 CIBER de Epidemiología y Salud Pública (CIBERESP), Barcelona, Spain

4 Department of Medicine, Universitat Internacional de Catalunya, Barcelona, Spain pneumococcal strains has been described during colonization $[4,5]$. Whether competition increases virulence is unknown.

To our knowledge, multiple serotype carriage has not been investigated during invasive pneumococcal disease (IPD).

\section{Methods}

\section{Patients and ethics}

We conducted a prospective study of children with IPD who were admitted to the pediatric Hospital Sant Joan de Déu in Barcelona, Spain, from January 2014 to December 2018. IPD was defined based on clinical diagnosis of the infection and culture isolation of pneumococci from a normally sterile body site.

Epidemiological data including age, gender, vaccination with PCV, antibiotic treatment and duration, days of hospitalization, and final diagnosis were recorded.

The study was approved by the Ethics and Clinical Research Committee of Hospital Sant Joan de Déu (Project PI16/00174).

\section{Detection of pneumococci in invasive samples}

Invasive samples were obtained from normally sterile body sites. Pneumococci were identified based on colony morphology, optochin susceptibility, and occurrence of $\alpha$-hemolysis 
on blood agar plates. Pure cultures were kept in skim milk, tryptone, glucose, glycerol medium (STGG) at $-80^{\circ} \mathrm{C}$.

\section{Detection of pneumococci and respiratory viruses in nasopharyngeal samples}

Nasopharyngeal aspirates were obtained by inserting a catheter, connected to a vacuum source, through one nostril until reaching the nasopharynx, applying suction and recovering the secretions into a sterile container. The procedure was repeated with the same catheter and container in the other nostril. The container was then washed with $3 \mathrm{~mL}$ of $1 \mathrm{x}$ phosphate-buffered saline (PBS), of which $1 \mathrm{~mL}$ was processed in the same day for culture and nucleic acid extraction, and $200 \mu \mathrm{L}$ was supplemented with $1 \mathrm{~mL}$ of STGG and kept at $-80^{\circ} \mathrm{C}$. The remaining volume was stored at $-80^{\circ} \mathrm{C}$. For seven patients, nasopharyngeal swabs were collected instead and inoculated in $1 \mathrm{~mL}$ of PBS.

Samples in STGG $(200 \mu \mathrm{L})$ were plated on gentamycin blood agar and incubated overnight at $37^{\circ} \mathrm{C}$ in anaerobiosis. Pneumococci were identified and isolated as described for invasive samples.

Total nucleic acid extraction was performed using the NucliSens easyMAG kit (BioMerieux Laboratories), according to the manufacturer's instructions, and used as template for real-time PCR. For pneumococcal detection, the presence of the lytA gene was investigated as described $[6,7]$. For respiratory viral detection, the multiplex real-time PCR Anyplex II RV16 detection kit (Seegene, South Korea), which targets 19 viruses (detailed in Table S1), was used.

\section{Pneumococcal serotyping}

Serotyping was determined by the Quellung reaction using specific antisera (Statens Serum Institute).

Molecular serotyping of lytA-positive nasopharyngeal samples was initially done at Hospital San Joan de Déu using fluorescence fragment analysis targeting 40 serotypes/ serogroups (detailed in Table S1) as previously described [6]. Upon sample arrival to Instituto de Tecnologia Química e Biológica, two additional approaches were used: real-time PCR targeting 23 serotypes/serogroups [6] and conventional multiplex PCR targeting 11 additional serotypes/serogroups (all detailed in Table S1) [8]. Non-typeable pneumococci were detected by real-time PCR or conventional PCR as described [9].

\section{Susceptibility to antibiotics}

Minimum inhibitory concentrations (MIC) to cefotaxime, penicillin (oral penicillin V), erythromycin, chloramphenicol, tetracycline, levofloxacin, and vancomycin were determined for invasive strains by agar dilution following Clinical and
Laboratory Standards Institute (CLSI) recommendations and interpretative criteria.

\section{Results}

Fifty-seven children diagnosed with IPD participated in the study. Demographic and epidemiological characteristics are detailed in Table 1. Briefly, $57.9 \%$ were less than 3 years old, $56.1 \%$ were males, and $57.9 \%$ had been vaccinated with at least one dose of a PCV. The most common diagnostics were pneumonia $(61.4 \%)$ and bacteremia $(22.8 \%)$.

Invasive samples were obtained mainly from blood $(82.5 \%)$ and over half $(59.6 \%)$ were collected on the same day of hospital admission (Table S1). The 57 pneumococcal invasive isolates belonged to 22 serotypes of which $24 \mathrm{~F}$ $(15.8 \%), 1(14.0 \%), 3(8.8 \%), 14(8.8 \%)$, and 19A (7.0\%) were the most prevalent. Twenty-five $(43.9 \%)$ isolates expressed serotypes covered by PCV13. Rates of nonsusceptibility to cefotaxime, penicillin, erythromycin, chloramphenicol, and tetracycline were $5.3 \%, 35.1 \%, 28.1 \%$, $1.8 \%$, and $29.8 \%$, respectively. All strains were susceptible to levofloxacin and vancomycin (Table S1).

The majority (61.4\%) of nasopharyngeal samples were obtained within 3 days of hospital admission. Of note, $26.3 \%$ $(n=15)$ were obtained before or within the first day of initiation of antibiotic treatment, $59.7 \%(n=34)$ were obtained between days $2-5$, and $14.0 \%(n=8)$ at a later period (Table S1, Figure S1).

Pneumococcal DNA was detected in approximately twothirds $(n=39,68.4 \%)$ of the nasopharyngeal samples. Pure cultures of pneumococci were obtained from ten of these samples. Notably, pneumococcal-negative samples were all, except one, collected at 2 days or more post-initiation of antibiotic treatment (Table S1, Figure S1).

Respiratory viruses were detected in $63.2 \%(n=36)$ of the children (Table 1). The most common were rhino/enterovirus (present in $31.6 \%$ of all samples), adenovirus (17.5\%), respiratory syncytial virus $(10.5 \%)$, and coronavirus $(8.8 \%)$ (Table 1 and Table S1). Thirteen (22.8\%) children had two or more viruses (Table S1). Half of the children (50.9\%) had both pneumococci and respiratory viruses.

Serotypes/serogroups were assigned to most $(34,87.2 \%)$ of the 39 nasopharyngeal samples positive for pneumococci. Although a serotype could not be determined for six samples (15.4\%), the capsular conserved gene cpsA was detected, suggesting that they contained pneumococci of serotypes not targeted by the molecular methods used (Table S1). As no viable cultures could be obtained from these samples (in agreement with the high $\mathrm{Ct}$ values obtained for $l y t A$, and the fact that they were obtained after initiation of antibiotic treatment), we could not unequivocally confirm the presence of pneumococci in them. In total, 23 serotypes were detected, of 
Table 1 Characteristics of the population

\begin{tabular}{ll}
\hline Patients information & No. $(\%)$ out of \\
& 57 \\
\hline$<3$ years old & $33(57.9)$ \\
Males & $32(56.1)$ \\
Vaccination with PCV & $33(57.9)$ \\
Age-appropriately vaccinated & $26\left(48.1^{\mathrm{b}}\right)$ \\
Antibiotic treatment before hospitalization & $9(15.8)$ \\
Fever before hospitalization & $54(94.7)$ \\
Diagnostic & \\
Pneumonia & $35(61.4)$ \\
Bacteremia & $13(22.8)$ \\
Septic arthritis & $4(7.0)$ \\
Meningitis & $4(7.0)$ \\
Sepsis & $1(1.8)$ \\
Respiratory viruses & \\
Rhino/enterovirus & $36(63.2)$ \\
Adenovirus & $18(31.6)$ \\
Respiratory syncytial virus & $10(17.5)$ \\
Coronavirus & $6(10.5)$ \\
Other & $5(8.8)$ \\
Pneumococcal carriage & $11(19.3)$ \\
Respiratory virus and pneumococcal carriage & $39(68.4)$ \\
Nasopharyngeal samples & $29(50.9)$ \\
Single serotype identical to invasive sample & $21(36.8)$ \\
Multiple serotypes including serotype identical to & $7(12.3)$ \\
invasive sample & \\
Single serotype different from invasive sample & $10(17.5)$ \\
Multiple serotypes different from serotype of invasive \\
$\quad$ sample & $1(1.8)$ \\
No pneumococcus detected & $18(31.6)$ \\
\hline &
\end{tabular}

${ }^{a}$ Vaccinated with at least one dose of any pneumococcal conjugate vaccine (PCV)

${ }^{\mathrm{b}}$ No information available on vaccination dates for 3 children as detailed in Table $\mathrm{S} 1$

${ }^{\mathrm{c}} 13$ children had more than one virus as detailed in Table $\mathrm{S} 1$

${ }^{\mathrm{d}}$ Other: parainfluenza 4 , fluA, fluB, bocavirus

which $3(12.8 \%), 19 \mathrm{~A}(12.8 \%), 1(10.3 \%), 14(7.7 \%)$, and $15 \mathrm{~A} / \mathrm{F}(7.7 \%)$ were the most prevalent (Table S1). Simultaneous detection of two serotypes occurred in $23.1 \%$ (9 of 39) of the nasopharyngeal pneumococcal-positive samples (Table 1, Table S1).

Comparison of pneumococcal findings in invasive and nasopharyngeal samples showed that in $28(49.1 \%)$ patients, the serotype found in the invasive source was also identified in the nasopharynx; among these, seven contained an additional serotype in the nasopharynx. Additionally, in $11(19.3 \%)$ patients, the serotype detected in the nasopharynx was different from the one detected in the invasive sample; for 18 patients (31.6\%), no pneumococci were detected in the nasopharynx. Together, in 18 of the 39 (46.2\%) nasopharyngeal samples where pneumococci were detected, a serotype different from the one identified in the invasive sample was found.

Interestingly, multiple serotype carriage was independent of the time elapsing from antibiotic treatment onset to nasopharyngeal sampling (Figure S1). Viral co-infection occurred similarly in nasopharyngeal samples with single or multiple serotypes ( $71.4 \%$ and $77.8 \%$, respectively, Table S1).

\section{Discussion}

We investigated serotype distribution in paired invasive and nasopharyngeal samples obtained from children during IPD. As pneumococcal infection is preceded by colonization, the strain (or serotype) recovered from a normally sterile body site should, in principle, be detected in the nasopharynx of the patient [1]. In our study, however, in around one-third (31.6\%) of the children, no pneumococci were detected. This was, most likely, due to the impact of ongoing antibiotic treatment at the time of nasopharyngeal sampling (Figure S1), resulting in low pneumococcal density or even clearance. To overcome this limitation, in future studies, nasopharyngeal sampling as close as possible to onset of disease, preferably before antibiotic treatment, would be desirable. Additionally, repeated sampling during disease might also provide additional information on the carriage dynamics during disease. Still, in half of the patients (49.1\%), a serotype match between the disease and the nasopharyngeal isolates was observed. Of particular interest, however, was the observation that a serotype different from the one causing disease was detected in $46.2 \%$ of all pneumococcal positive samples. These observations suggest that multiple serotype carriage (or co-colonization) is very frequent among these patients, and higher than previously estimated for healthy children living in Europe (20$30 \%)[3,4]$.

Of interest, we were able to detect serotype 1 in the nasopharyngeal samples of 4 of the 8 patients with IPD caused by this serotype. Serotype 1 is an important cause of IPD, has been associated with outbreaks, and is notorious for being infrequent in colonization [10]. Recent studies have shown that this is partly explained by a short duration of carriage associated with a high invasiveness and rapid dissemination [11]. The latter has been linked to a high cytotoxicity due to release of pneumolysin upon high levels of bacterial lysis [12].

Previous studies have shown that competition for nutrients and space occurs between pneumococcal strains during colonization [5]. This phenomenon is mediated, at least in part, by bacteriocin production. Whether competition may increase the virulence of the excluded strain remains to be tested.

In our study, nearly all children had fever at the onset of infection and respiratory viruses were detected in around 60\% of them. Respiratory viruses are known to increase 
pneumococcal density in the nasopharynx and the likelihood of pneumococcal infection [13,14]. Notably, it has been shown that fever leads to increased shedding of pneumococcal cells from biofilm and these cells have altered gene expression patterns that result in increased virulence [15].

Taken together, we hypothesize that the presence of viruses and of pneumococcal competition derived from multiple serotype carriage might increase virulence of some pneumococcal cells and, ultimately, the likelihood of an infection. However, as this was an observational study, with a limited number of samples, additional studies designed to address this hypothesis are needed. Such studies could compare carriage in patients with IPD with and without viral co-infection.

To our best knowledge, this is the first report describing high frequency of pneumococcal multiple serotype carriage in children with IPD. Whether competition during multiple serotype carriage is important for the onset and progress to disease warrants further investigation.

Supplementary Information The online version contains supplementary material available at https://doi.org/10.1007/s10096-021-04231-4.

Acknowledgements The authors thank all the pediatricians and staff of the Hospital Sant Joan de Déu, parents, and children who participated in the study. We also thank National Center for Microbiology of Majadahonda (Madrid, Spain) for antimicrobial susceptibility testing.

Author contribution CMA and RSL contributed to the concept and design of the study. CMA and RSL contributed with reagents and materials. CMA contributed with samples. Data acquisition was done by DH and SF. Data analysis and interpretation of results was done by SF, CMA, and RSL. The manuscript was drafted by SF and RSL. All authors have critically revised the manuscript and approved the final version.

Funding This work was supported by projects LISBOA-01-0145FEDER (Microbiologia Molecular, Estrutural e Celular), funded by the European Regional Development Fund (FEDER) through COMPETE2020 - Programa Operacional Competitividade e Internacionalização); LISBOA-01-0145-FEDER-016417 (ONEIDA cofunded by Fundos Europeus Estruturais e de Investimento, Programa Operacional Regional Lisboa 2020; and Fundação para a Ciência e a Tecnologia (FCT)), and PI16/00174, co-funded by Plan Nacional I+D+ I, ISCIII-Subdirección General de Evaluación y Fomento de la Investigación Sanitaria; FEDER; and the Catalan Agency for the Management of Grants for University Research AGAUR (grant 2017 SGR 0742). SF was supported by FCT (grant PD/BD/105732/2014). DH was supported by Instituto de Salud Carlos III (grant FI17/00248).

Data availability Not applicable.

\section{Declarations}

Ethics approval The study was approved by the Ethics and Clinical Research Committee of Hospital Sant Joan de Déu (Project PI16/00174).

Consent for publication Patients' data was analyzed anonymously.

Conflict of interest The authors declare no competing interests.

\section{References}

1. Gerlini A, Colomba L, Furi L, Braccini T, Manso AS, Pammolli A, Wang B, Vivi A, Tassini M, van Rooijen N, Pozzi G, Ricci S, Andrew PW, Koedel U, Moxon ER, Oggioni MR (2014) The role of host and microbial factors in the pathogenesis of pneumococcal bacteraemia arising from a single bacterial cell bottleneck. PLoS Pathog 10(3):e1004026

2. Ganaie F, Saad JS, McGee L, van Tonder AJ, Bentley SD, Lo SW, Gladstone RA, Turner P, Keenan JD, Breiman RF, Nahm MH (2020) A new pneumococcal capsule type, 10D, is the 100th serotype and has a large cps fragment from an oral streptococcus. $\mathrm{mBio}$ $11(3)$

3. Wyllie AL, Wijmenga-Monsuur AJ, van Houten MA, Bosch AATM, Groot JA, van Engelsdorp GJ, Bruin JP, Bogaert D, Rots NY, Sanders EAM, Trzciński K (2016) Molecular surveillance of nasopharyngeal carriage of Streptococcus pneumoniae in children vaccinated with conjugated polysaccharide pneumococcal vaccines. Sci Rep 6:23809

4. Valente C, Hinds J, Gould KA, Pinto FR, de Lencastre H, Sá-Leão $\mathrm{R}$ (2016) Impact of the 13-valent pneumococcal conjugate vaccine on Streptococcus pneumoniae multiple serotype carriage. Vaccine 34(34):4072-4078

5. Shen P, Lees JA, Bee GCW, Brown SP, Weiser JN (2019) Pneumococcal quorum sensing drives an asymmetric ownerintruder competitive strategy during carriage via the competence regulon. Nat Microbiol 4(1):198-208

6. Selva L, del Amo E, Brotons P, Muñoz-Almagro C (2012) Rapid and easy identification of capsular serotypes of Streptococcus pneumoniae by use of fragment analysis by automated fluorescence-based capillary electrophoresis. J Clin Microbiol 50(11):3451-3457

7. Carvalho MG, Tondella ML, McCaustland K, Weidlich L, McGee L, Mayer LW, Steigerwalt A, Whaley M, Facklam RR, Fields B, Carlone G, Ades EW, Dagan R, Sampson JS (2007) Evaluation and improvement of real-time PCR assays targeting lytA, ply, and psaA genes for detection of pneumococcal DNA. J Clin Microbiol 45(8): 2460-2466

8. Pai R, Gertz RE, Beall B (2006) Sequential multiplex PCR approach for determining capsular serotypes of Streptococcus pneumoniae isolates. J Clin Microbiol 44(1):124-131

9. Tavares DA, Handem S, Carvalho RJ, Paulo AC, de Lencastre H, Hinds J, Sá-Leão R (2019) Identification of Streptococcus pneumoniae by a real-time PCR assay targeting SP2020. Sci Rep 9(1):3285

10. Almeida ST, de Lencastre H, Sá-Leão R (2013) Epidemiology and population structure of serotypes 1,5 and $7 \mathrm{f}$ carried by children in Portugal from 1996-2010 before introduction of the 10-valent and 13-valent pneumococcal conjugate vaccines. PLoS One 8(9): e75442

11. Chaguza C, Senghore M, Bojang E, Lo SW, Ebruke C, Gladstone RA, Tientcheu PE, Bancroft RE, Worwui A, Foster-Nyarko E, Ceesay F, Okoi C, McGee L, Klugman KP, Breiman RF, Barer MR, Adegbola RA, Antonio M, Bentley SD, Kwambana-Adams BA (2020) Carriage dynamics of pneumococcal serotypes in naturally colonized infants in a rural african setting during the first year of life. Front Pediatr 8:587730

12. Jacques LC, Panagiotou S, Baltazar M, Senghore M, Khandaker S, Xu R, Bricio-Moreno L, Yang M, Dowson CG, Everett DB, Neill DR, Kadioglu A (2020) Increased pathogenicity of pneumococcal serotype 1 is driven by rapid autolysis and release of pneumolysin. Nat Commun 11(1):1892

13. Fan RR, Howard LM, Griffin MR, Edwards KM, Zhu Y, Williams JV, Vidal JE, Klugman KP, Gil AI, Lanata CF, Grijalva CG (2016) 
Nasopharyngeal pneumococcal density and evolution of acute respiratory illnesses in young children, Peru, 2009-2011. Emerg Infect Dis 22(11):1996-1999

14. Murad C, Dunne EM, Sudigdoadi S, Fadlyana E, Tarigan R, Pell CL, Watts E, Nguyen CD, Satzke C, Hinds J, Dewi MM, Dhamayanti M, Sekarwana N, Rusmil K, Mulholland EK, Kartasasmita C (2019) Pneumococcal carriage, density, and cocolonization dynamics: a longitudinal study in Indonesian infants. Int J Infect Dis 86:73-81
15. Pettigrew MM, Marks LR, Kong Y, Gent JF, Roche-Hakansson H, Hakansson AP (2014) Dynamic changes in the Streptococcus pneumoniae transcriptome during transition from biofilm formation to invasive disease upon influenza A virus infection. Infect Immun 82(11):4607-4619

Publisher's note Springer Nature remains neutral with regard to jurisdictional claims in published maps and institutional affiliations. 